\title{
Avaliação DA MATURIDAde FETAL EM GESTAÇÕES DE ALTO RISCO: ANÁLISE DOS RESULTADOS DE ACORDO COM A IDADE GESTACIONAL
}

R.M.Y. Nomura*, S. Miyadahira, R.P.V. Francisco, D. Okatani, M. Zugaib

SetordeVitalidadeFetal ClínicaObstétricado Hospital das Clínicas da FaculdadedeMedicinadaUniversidadedeSãoPaulo

RESUMO - OBjETIVO. Estudar a avaliação da maturidade fetal em gestaçōes de alto risco e analisar os resultados neonatais.

Métodos. Entre julho de 1998 e agosto de 1999 foram realizadas, no Setor de Vitalidade Fetal da Clínica Obstétrica do HCFMUSP, 180 amniocenteses para avaliação da maturidade fetal, sendo realizados os testes de Clements em três tubos e a contagem de células orangiófilas coradas com Azul de Nilo a $0,1 \%$. Os resultados perinatais foram correlacionados com a maturidade fetal em 75 casos cujo parto ocorreu até sete dias após a punção.

RESULTADOS. Na macroscopia, $91 \%$ das amostras apresentavam líquido amniótico claro, 3,3\% meconial e 5,6\% hemorrágico. A maturidade foi observada em $28 \%$ dos exames realizados. $\mathrm{Na}$ avaliação dos resultados perinatais, quando a maturidade estava ausente, a necessidade de intubação do recém-nascido ocorreu em três casos (13\%) e nos fetos maduros isto ocorreu em um caso $(2,5 \%)(p<0,05)$. A necessidade de internação em UTI neonatal ocorreu em $65 \%$ dos recém-nascidos que apresentavam líquido imaturo e em apenas $10 \%$ dos maduros $(p<0,0001)$.

CONCLUSÖES. Os recém-nascidos das gestantes com maturidade fetal presente apresentaram com menor freqüência necessidade de intubação na sala de parto e de internação em UTI neonatal, demonstrando menor morbidade perinatal. Entre a $29^{\mathrm{a}}$ e a $32^{\mathrm{a}}$ semana de gestação, foram observados cerca de $10 \%$ de fetos maduros, demonstrando que, quando necessário, a avaliação da maturidade pode ser realizada neste período da gravidez. Não observamos casos com maturidade fetal abaixo de 29 semanas, limitando a realização deste exame neste período.

UNITERMOS: Maturidadefetal.Amniocentese. Líquido amniótico.

\section{INTRODUÇÃO}

Amaturidadefetalconsistenoplenodesenvolvimento dos diversos órgãos e sistemas fetais que, noseu processo fisiológiconormal, se completa entre 37 e 40 semanas de gestação.Amaturidade dosistemarespiratóriofetal ocorreao redor da $35^{\mathrm{a}}$ semana de gestação, quandoasadaptaçõesanatômicas efuncionais permitemaorecém-nascido(RN)prematuro sobreviveraoambienteextra-uterino ${ }^{1,2}$.

É conhecido quealgumas condições clí-

*Correspondência:

Rua General Canavarro, 280 - Bairro Campestre

- Santo André - SP - CEP 09070-440

Tel: 49912481 (res) - 42218778(com)

FAX: 49917312(res) - 42218752(com)

Home Page: http://www.honet.usp.br/ob/ nicas aceleramamaturidadefetal (porexemplo a hipoxia fetal crônica), enquanto que outras, como o diabete melito, estão associadas a umatraso na maturação pulmonar. A avaliação da maturidade pulmonar fetal, através daanálise do líquidoamniótico(LA), pode ser realizada antes da resolução da gestação, principalmentenaquelas pré-termo, evitando-se assim, em algumas situações, oparto prematuro iatrogênico. Entre as múltiplas complicações da prematuridade, a imaturidade pulmonar, relacionada à produção inadequada de surfactante, constituia de maior gravidade, comprometendo, muitas vezes, a sobrevida do concepto ${ }^{3}$.

O diagnóstico de maturidade pulmonar fetal através da análise do líquido amniótico apresentaincidência variável de acordo com a idade gestacional em que a amostra é coletada ${ }^{4}$. É importante tambéma avaliação das doenças maternas e das intercorrências obstétricas associadas. Deste modo, os objetivos deste estudo foram avaliar os resultados dapesquisa dematuridade fetal, em gestações dealto risco, através da análise de líquido amniótico obtido por amniocentese, analisando o comportamento destes resultados de acordo com aidade gestacional na data da punção.

\section{Métodos}

Durante o período de julho de 1998 a agosto de 1999 foram realizadas $180 \mathrm{am}$ niocenteses em gestantes de alto risco, acompanhadas pelo Setor de Vitalidade 
Fetal da Clínica Obstétrica do Hospital das Clínicas da Faculdade de Medicina da Universidade de São Paulo. Estas pacientes oriundas tanto do ambulatório como da enfermaria da Clínica Obstétrica, foram encaminhadas ao setor para a coleta do líquido amniótico e avaliação da vitalidade fetal. As provas de maturidade fetal foram realizadas pela equipe do setor de vitalidade, imediatamente após a coleta do líquido. Os resultados obtidos foram armazenados prospectivamente no banco de dados desenvolvido peloSetor. A conduta obstétrica variou de acordo com agravidade do quadro clínico materno e fetal. Os resultados neonataisforam coletadosa partir da consultado livro de registro de nascimentos.

Para a análise das freqüências, foram incluídos todos os exames realizados no $L A$, independentemente do parto ter sido realizado ou não neste hospital. Na análise dos resultados perinatais, foram excluídas as pacientes que não realizaram o parto nesta instituição, sendo estudados somente os casos em que a amniocentese ocorreu no máximo sete dias antes da data do parto.

Os métodos laboratoriais utilizados na avaliação da maturidade fetal basearam-sena análise do líquido amniótico obtido durantea gestação, através da amniocentese. Foram avaliados os aspectos macroscópicos, microscópicos e o teste de Clements.

Aavaliaçãomacroscópica das características do líquido amniótico foi realizadaimediatamente após a coleta. De acordo com o aspecto do LA, estepôdeser classificadoem: claro, hemorrágico, meconial ou amarelado (esteúltimoocorrendogeralmenteemgestaçõesmuitoprematuras). Foi observado também a presença ou não de grumos. Quando os grumos estavam presentes, estes foram ainda classificados em finos, médios ou grossos(líquido opalescente).
A análise pela microscopia óptica foi realizada pela Citologia com Azul de Nilo ou índice cito-lipídico. Esta técnica consiste na mistura de uma gota de LA com uma gota de sulfato Azul de Nilo a 0,1\%, homogeneização e leitura em microscópio ótico comum. As células ricas em gorduras coram-se de laranja, sendo denominadas células orangiófilas (células da maturidade). Até a $34^{\mathrm{a}}$ semana observase menos de $1 \%$ de células orangiófilas, entre 34 e 38 semanas encontramos $1 \%$ a $10 \%$ destas células, entre 38 e 40 semanas de $10 \%$ a $50 \%$ e acima de 40 semanas acima de $50 \%$. Estas células são de origem descamativa, provenientes da epiderme fetal, sendo células da superfície queratinizada do epitélio, revestido de gordura das glândulas sebáceas. Portanto, este teste avalia a maturidade da pele fetal. Morrison et al. (1978) ${ }^{4}$ sugerem que a maturidade da pele ocorra ao mesmo tempo que a maturidade pulmonar fetal.

$O$ teste de Clements foi realizado imediatamente após a coleta do líquido amniótico. Este método proposto por Clements et al. $(1972)^{5}$ se baseia na capacidade do sistema surfactante de formar bolhas estáveis na interface arlíquido, na presença de etanol. As bolhas serão mais estáveis quanto maior a quantidade de substâncias surfatantes no LA. Utilizou-se de três tubos limpos de 8 a $14 \mathrm{~mm}$ de diâmetro por $100 \mathrm{~mm}$ de altura, colocando-se em cada tubo as seguintes quantidades de LA: $1,0 \mathrm{ml}$, $0,75 \mathrm{ml}$ e $0,50 \mathrm{ml}$. Completou-se o volume para I,0 ml com soro fisiológico. Em seguida, adicionou-se $1 \mathrm{ml}$ de etanol a $95 \%$ em todos os tubos, sendo fechados e agitados vigorosamente cada um destes tubos por 15 segundos e a leitura foi feita após um período de 15 minutos. O teste foi considerado positivo quando se verificou a presença de anel completo de bolhas; estáveis até o terceiro tubo; negativo quando não se verificou a presença de bolhas, e intermediário quando as bolhas não se completaram até o terceiro tubo. Este teste pode provocar resultados falso-positivos quando o LA apresentar-se contaminado de sangue ou mecônio.

Para se obter um laudo final a respeito da avaliação da maturidade fetal, utilizou-se uma classificação baseada nos resultados obtidos. Foi considerado como maturidade fetal presente quando a contagem de células orangiófilas foi superior ou igual a 10\% e/ou teste de Clements positivo nos três tubos; maturidade intermediária quando a contagem de células orangiófilas estava entre $6 \%$ a $9 \%$ e/ou teste de Clements positivo até $\circ 2^{\circ}$ tubo; e maturidade ausente quando a contagem de células orangiófilas foi inferior ou igual a $5 \%$ e teste de Clements positivo apenas no primeiro tubo ou negativo nos três tubos.

Foram analisados somente os resultados neonatais dos casos cujo parto ocorreu nesta instituição, sendo avaliados os seguintes parâmetros: tipo de parto, índices de Apgar de $1^{\circ}$ e $5^{\circ}$ minutos, peso do recém-nascido (RN), incidência de $R N$ pequenos para a idade gestacional (PIG) e a internação em UTI neonatal. Os RN foram classificados como PIG quando o peso de nascimento foi inferior ao percentil 10 para a idade gestacional, de acordo com a curva de normalidade desenvolvida pelo setor de neonatologia deste hospital.

Aidade gestacional foi calculada a partir da data da última menstruação (DUM) quando esta foi compatível com exame 
ultra-sonográfico realizado até a $20^{\mathrm{a}}$ semana. Quando a paciente não soube referir a DUM ou na discordância com o exame ultra-sonográfico, a datação da gestação foi realizada com base na primeira ultra-sonografia realizada.

Foram analisadas as freqüências dos resultados dos exames e para a comparação entre os grupos de estudo utilizouse do teste do Qui Quadrado para as variáveis qualitativas, com correção de Yates para continuidade e, quando pertinente, o teste Exato de Fisher. Na avaliação das variáveis quantitativas, utilizouse do teste $t$ de student. Adotou-se como nível de significância o valor 0,05 $(\propto=5 \%)$. Com isso, níveis descritivos (p) inferiores a esse valor foram considerados significantes $(p<0,05)$.

\section{RESUltados}

Foram realizadas 180 amniocenteses em 145 gestantes de alto risco pelo Setor de Vitalidade Fetal no período entre 01/07/98 e 31/08/99. A idade média das pacientes foi de 29,3 anos, com desvio padrão de 6,8 anos. Em relação aos antecedentes obstétricos, 43 pacientes $(29,7 \%)$ eram primigestas, $34(23,4 \%)$ apresentavam duas gestações anteriores e $68(46,9 \%)$ apresentavam três ou mais gestações anteriores. Cinqüenta e sete pacientes $(39,3 \%)$ eram nulíparas, $40(27,6 \%)$ apresentavam um parto anterior, e 48 $(33,1 \%)$ referiam dois ou mais partos anteriores. As principais intercorrências clínicas ou obstétricas foram: síndromes hipertensivas em 80 casos $(55,2 \%)$, diabetes em 19 casos $(13,1 \%)$, crescimento intra-uterino retardado (CIUR) em I I $\operatorname{casos}(7,6 \%)$ e cardiopatia materna em 10 casos $(6,9 \%)$.

\section{Figura I - Distribuição da população em relação ao número de amniocenteses realizadas por paciente}

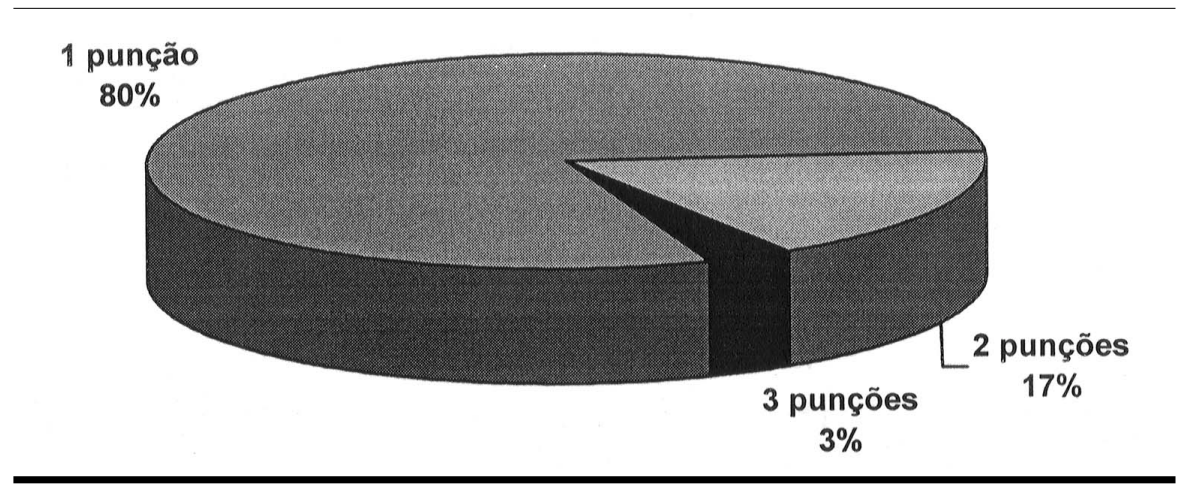

Tabela I - Distribuição dos casos de acordo com o aspecto macroscópico do líquido amniótico nas faixas de idade gestacional

\begin{tabular}{ccccc}
\hline $\begin{array}{c}\text { Idade } \\
\text { gestacional } \\
\text { (semanas) }\end{array}$ & $\mathbf{n}$ & Claro & Meconial & Hemorrágico \\
\hline 27 & 3 & $2(66,6 \%)$ & $\mathrm{I}(33,3 \%)$ & $0(0 \%)$ \\
28 & 3 & $3(100 \%)$ & $0(0 \%)$ & $0(0 \%)$ \\
29 & 11 & $9(81,8 \%)$ & $\mathrm{I}(9,1 \%)$ & $\mathrm{I}(9,1 \%)$ \\
30 & 10 & $9(90,0 \%)$ & $0(0 \%)$ & $\mathrm{I}(10,0 \%)$ \\
31 & 12 & $10(83,3 \%)$ & $\mathrm{I}(8,3 \%)$ & $\mathrm{I}(8,3 \%)$ \\
32 & 17 & $16(94,1 \%)$ & $\mathrm{I}(5,9 \%)$ & $0(0 \%)$ \\
33 & 22 & $21(95,5 \%)$ & $0(0 \%)$ & $1(4,5 \%)$ \\
34 & 23 & $19(82,6 \%)$ & $1(4,3 \%)$ & $3(13,4 \%)$ \\
35 & 35 & $33(94,2 \%)$ & $1(2,9 \%)$ & $1(2,9 \%)$ \\
36 & 21 & $21(100 \%)$ & $0(0 \%)$ & $0(\%)$ \\
$\geq 37$ & 23 & $21(91,3 \%)$ & $0(0 \%)$ & $2(8,7 \%)$ \\
Total & 180 & $164(91,1 \%)$ & $6(3,3 \%)$ & $10(5,6 \%)$ \\
\hline
\end{tabular}

Na figura I, podemos observar a distribuição da população analisada em relação ao número de amniocenteses realizadas por paciente. Cercade $80 \%$ da populaçãoestudada necessitou de apenas uma amniocentese, e em somente 5 (3,5\%) houve necessidade de realizar em três ocasiões a coleta dolíquido amniótico.

Em relação aos aspectos macroscópicos do líquido amniótico, podemos observar na tabela I a distribuição dos casos nas diferentes faixas de idade gestacional. $\mathrm{Na}$ tabela 2está demonstradaa distribuição dos casos deacordo com os resultados do teste de Clements e da contagem de células orangiófilas, nas diversas faixas de idade gestacional, bem como a interpretação da maturidade fetal. Em dois casos de líquido amniótico meconial e em quatro de líquido hemorrágico, a interpretação dos testes não foi conclusiva, prejudicando a avaliação da maturidadefetal. 
Tabela 2 - Distribuição dos casos de acordo com a maturidade fetal e os resultados do teste de Clements e da contagem de células orangiófilas nas diversas faixas de idade gestacional

\begin{tabular}{|c|c|c|c|c|c|c|c|c|c|c|c|c|}
\hline \multirow{2}{*}{$\begin{array}{c}\text { IG } \\
\text { semanas }\end{array}$} & \multicolumn{4}{|c|}{ TESTE DE CLEMENTS } & \multicolumn{2}{|c|}{ CÉLULAS } & \multicolumn{2}{|c|}{ ORANGIÓFILAS } & \multicolumn{4}{|c|}{ INTERPRETAÇÃO } \\
\hline & $n$ & Pos Itb & Pos. 2tb & Pos 3tb & $n$ & $\leq 5 \%$ & $5-9 \%$ & $\geq 10 \%$ & $\mathbf{n}$ & Maduro & Intermediár & - Imaturo \\
\hline 27 & 3 & $I(33,3 \%)$ & 0 & 0 & 2 & $2(100 \%)$ & 0 & 0 & 3 & 0 & 0 & $3(100 \%)$ \\
\hline 28 & 3 & 0 & 0 & 0 & 3 & $3(100 \%)$ & 0 & 0 & 3 & 0 & 0 & $3(100 \%)$ \\
\hline 29 & 10 & 0 & $\mathrm{I}(\mathrm{I0,0 \% )}$ & $2(20,0 \%)$ & 8 & $6(75,0 \%)$ & $2(25,0 \%)$ & 0 & ॥ & $2(18,2 \%)$ & $2(18,2 \%)$ & $7(63,6 \%)$ \\
\hline 30 & 10 & 0 & 0 & $\mathrm{I}(10,0 \%)$ & 8 & $8(100 \%)$ & 0 & 0 & 10 & $\mathrm{I}(10 \%)$ & 0 & $9(90 \%)$ \\
\hline 31 & II & $2(18,2 \%)$ & $2(18,2 \%)$ & $2(18,2 \%)$ & 10 & $9(90,0 \%)$ & $\mathrm{I}(10,0 \%)$ & 0 & II & $\mid(9,1 \%)$ & $2(18,2 \%)$ & $8(72,7 \%)$ \\
\hline 32 & 17 & $3(17,6 \%)$ & $3(17,6 \%)$ & 0 & 16 & $\mid 4(87,5 \%)$ & $2(12,5 \%)$ & 0 & 17 & 0 & $3(17,6 \%)$ & $14(82,3 \%)$ \\
\hline 33 & 21 & 0 & $3(14,3 \%)$ & $6(28,6 \%)$ & 18 & $8(44,4 \%)$ & $5(27,8 \%)$ & $5(27,8 \%)$ & 21 & $7(33,3 \%)$ & $3(14,3 \%)$ & II (52,4\%) \\
\hline 34 & 20 & $3(15,0 \%)$ & $6(30,0 \%)$ & $2(10,0 \%)$ & 15 & $10(66,7 \%)$ & I (6,7\%) & $4(26,7 \%)$ & 22 & $5(22,7 \%)$ & $3(13,6 \%)$ & $14(63,6 \%)$ \\
\hline 35 & 34 & $6(17,6 \%)$ & $7(20,6 \%)$ & $9(26,5 \%)$ & 27 & II (40,7\%) & $7(25,9 \%)$ & $9(33,3 \%)$ & 34 & II $(32,3 \%)$ & $7(20,6 \%)$ & $16(47,1 \%)$ \\
\hline 36 & 21 & $3(\mid 4,3 \%)$ & $5(23,8 \%)$ & $7(33,3 \%)$ & 16 & $7(43,7 \%)$ & $5(31,2 \%)$ & $4(25,0 \%)$ & 21 & $7(33,3 \%)$ & $3(14,3 \%)$ & II (52,4\%) \\
\hline$\geq 37$ & 21 & $2(9,5 \%)$ & $2(9,5 \%)$ & $14(66,7 \%)$ & II & $3(27,3 \%)$ & 0 & $8(72,7 \%)$ & 21 & $15(7 \mid, 4 \%)$ & $2(9,5 \%)$ & $4(19,1 \%)$ \\
\hline
\end{tabular}

Total $\quad 17120(11,7 \%) 30(17,5 \%) 43(25,2 \%)$

|348I (60,4\%)23 (17,2\%)30 (22,4\%) I7449 (28,2\%) $25 \quad(14,4 \%) 100 \quad(57,4 \%)$

Tabela 3 - Distribuição dos casos quanto às características macroscópicas, teste de Clements (TC) e contagem de células orangiófilas (CO) no líquido amniótico (LA), de acordo com a avaliação da maturidade fetal

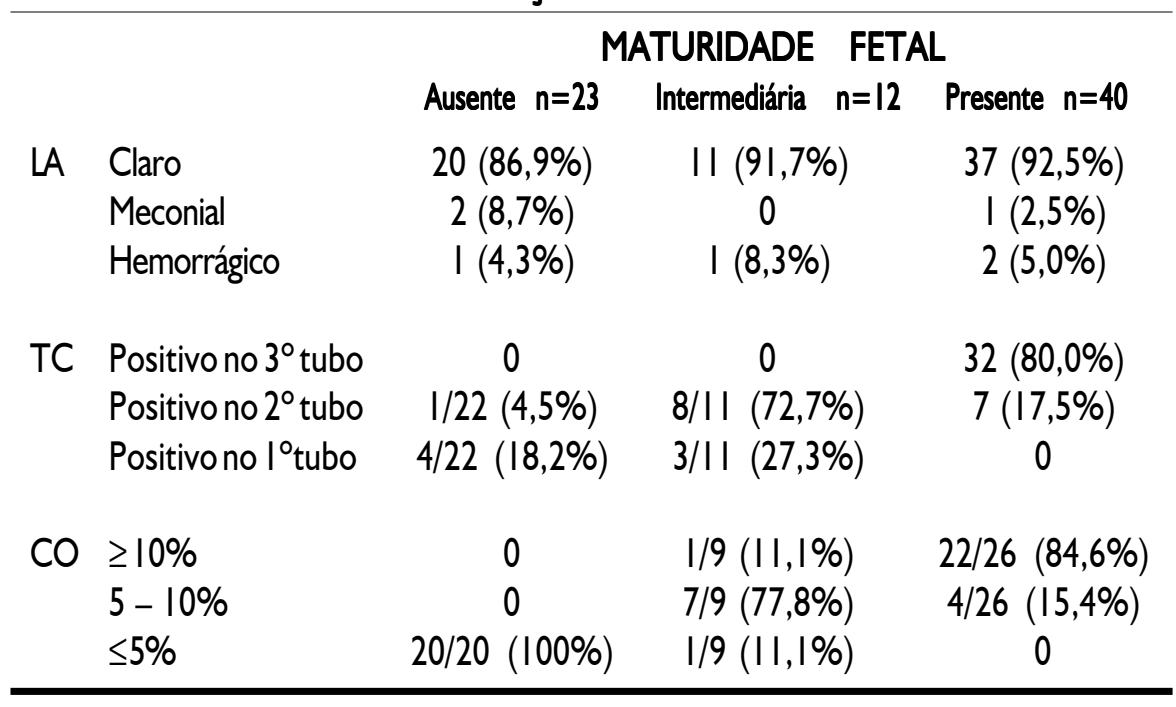

Em I4 pacientes com diagnóstico de Diabetes, não associado à sindrome hipertensiva, foram realizadas 15 amniocenteses. Entre sete exames realizados em gestações acima de 37 semanas, apenas um apresentou LA imaturo ( $14,3 \%)$. Oito amniocenteses foram realizadas antes da $37^{\mathrm{a}}$ semana, eneste grupo quatro apresentaram LAma- duro (50\%), dois intermediários (25\%) e dois imaturos $(25 \%)$.

Para o estudo dos resultados perinatais foram selecionadas 75 pacientes, cujaúltima amniocentese foi realizada no máximo sete dias antes da data do parto. Na tabela 3, podemos observar a distribuição dos casos quanto às características macroscópicas, teste de Clements e contagem de células orangiófilas no líquidoamniótico, de acordo com a avaliação da maturidade fetal. $\mathrm{Na}$ tabela4, estão demonstrados os resultados perinatais de acordo com a avaliação da maturidade fetal nestes casos. Podemos observar que quando a maturidade estava presente, houve uma menor necessidade de intubação do recém-nascido em sala de parto e de internação dos mesmos em unidades de terapia intensiva neonatais.

\section{Discussão}

Os testes de avaliação da maturidade fetal sofrem influências de diversosfatores, 
Nomura RMY etal.

\begin{tabular}{|c|c|c|c|}
\hline \multicolumn{4}{|c|}{$\begin{array}{c}\text { Tabela } 4 \text { - Resultados perinatais de acordo com a avaliação da maturidade fetal } \\
\text { realizada até } 7 \text { dias antes da data do parto }\end{array}$} \\
\hline & Ausente $n=23$ & $\begin{array}{ll}\text { MATURIDADE } & \text { FETAL } \\
\text { Intermediária } & n=12\end{array}$ & Presente $n=40$ \\
\hline IG (sem, média \pm DP) & $33,01 \pm 2,77$ & $34,78 \pm 2,36$ & $35,52 \pm 2,43$ \\
\hline Peso do RN (g, média \pm DP) & $1658,04 \pm 668,04$ & $2135,01 \pm 828,63$ & $2325,25 \pm 829,77$ \\
\hline RN PIG & $3(13,0 \%)$ & $5(4 I, 7 \%)$ & $8(20,0 \%)$ \\
\hline Índice de Apgar de $1^{\circ} \min <7$ & $9(39,1 \%)$ & $5(4 I, 7 \%)$ & $9(22,5 \%)$ \\
\hline Índice de Apgar de $5^{\circ} \mathrm{min}<7$ & $2(8,7 \%)$ & $0(0 \%)$ & $0(0 \%)$ \\
\hline Intubação do RN na sala de parto & $3(\mid 3,0 \%)$ & $3(25,0 \%)$ & | $(2,5 \%)^{*}$ \\
\hline UTIneonatal & $15(65,2 \%)$ & $6(50,0 \%)$ & $4(10,0 \%)^{* *}$ \\
\hline${ }^{*} \mathrm{p}<0,05 *{ }^{*} \mathrm{p}<0,0001$ & & & \\
\hline
\end{tabular}

entre eles a contaminação com sangue ou mecônio, da coleta e armazenamento, e da técnica utilizada na sua avaliação. Os resultados falso-positivos são muito raros ea taxa de falso-negativos oscila entre $8 \%$ a $40 \%$. Torna-se necessária a avaliação do LA através de diferentes técnicas para assegurar um melhor resultado, principalmente nas gestações longe do termo. Novas metodologias têm sido propostas para oestudo do líquido amniótico, porém, freqüentemente, a utilização destas metodologias promovem um aumento nos custos. Assim, o teste de Clements e o estudo das células orangiófilas constituem exames de baixíssimo custo, quando comparados aos tradicionais testes como o estudo da relação lecitina/esfingomielina por cromatografia em camada delgadanoLA 6 .

Atualmente, com os avanços da perinatologia e da terapia intensiva neonatal, a pesquisa da maturidade fetal através do LA tornou-se importante, principalmente nas gestações de alto risco longe do termo, quando devemos ponderar os benefícios do parto prematuro frente à condição da maturidade pulmonar fetal. Na nossa casuística, podemos constatar que, após a $29^{\mathrm{a}}$ semana de gestação, a maturidade pulmonar pode estar presente, e de acordo com a gravidade do quadro clínico materno ou fetal, a opção de resolução da gestação é muito oportuna, uma vez que se confirme a maturidade fetal. É claro que, por ser a amniocentese um procedimento invasivo, não isento de riscos para a mãe e para o concepto, devemos ponderar sempre o real benefício do procedimento. A incidência de 5,6\% de líquido amniótico hemorrágico, sugerindo um sangramento durante a punção, deve sempre ser considerado. No Setor de Vitalidade Fetal, realizamos a amniocentese sob visão ultra-sonográfica, e evitamos a punção transplacentária. Somente em casos especiais este tipo de punção é realizada, sempre conscientes dos riscos existentes. Quando é evidenciado a coleta de líquido amniótico hemorrágico, preconizamos como rotina a visibilização do local puncionado para eventual constatação ultra-sonográfica de sangramento ativo na cavidade amniótica. Estes episódios de sangramento contínuo não devem apresentar duração superior a três minutos, caso contrário a possibilidade da realização da cesariana deve ser cogitada. Cessando o sangramento, é imprescindível a monito- rização da freqüência cardíaca fetal por pelo menos 20 minutos, através da cardiotocografia. A ocorrência de alterações no traçado poderão evidenciar o sofrimento fetal.

Outro aspecto importante de ser abordado é a detecção do líquido amniótico meconial, indicando a resolução da gestação. A eliminação de mecônio pelo feto, duranteavidaintra-uterina, freqüentemente acompanha os casos dehipoxemiafetal, que pode ocorrer nos casos de hipertensão arterial, crescimento intra-uterino restrito e na vigência do oligohidrâmnio ${ }^{7,8}$. No acompanhamento de gestantes de alto-risco, na nossa casuística, em $33 \%$ foi evidenciado o líquido meconial, demonstrandoagravidade destes casos.

Ao avaliarmos os resultados perinatais, observamos que os recém-nascidos das gestantes com maturidadefetal presentena avaliação do líquidoamnióticonasemanaanterior ao parto, apresentaram commenorfreqüêncianecessidade de intubação na sala de parto e de internação em UTI neonatal, demonstrando menor morbidade perinatal. Estas constatações facilitama tomada de decisões frentea casos graves do ponto de vista materno, quandoa maturidade fetal estiverpresente, mesmo longe do termo dagestação. Po- 
rém, aimaturidadefetal nos incentivaaadotar condutas conservadoras, na tentativa de se prolongar ao máximoa gestação, para queo fetoatinjaalgumgrau dematuridadepulmonar antes do nascimento. Entre a $29^{\mathrm{a}}$ e a $32^{\mathrm{a}}$ semanadegestação, foramobservadoscerca de $10 \%$ de fetos maduros, demonstrando que, quando necessário, aavaliação da maturidade pode ser realizada neste período da gravidez. Não observamos casos com maturidade fetal abaixo de 29 semanas, limitando arealização desteexameneste período.

\section{Conclusões}

Este estudo nos permite concluir que, nas situações pertinentes onde a resolução dagestação éimportante para amelhora do quadro materno ou fetal, a amniocentese para coleta de líquido amniótico tem o seu valor, uma vez que a constatação da maturidade fetal tranqüiliza a mãe e o obstetra quanto à realização do parto prematuro terapêutico. Arealização de amniocentese em gestações inferiores a 29 semanas deve sempre ser discutida, pois, na nossa casuística, os exames realizados nesta faixa nãoforam capazes de diagnosticar maturidade fetal. Quando a maturidade fetal está presente na análise do líquido amniótico, ocorre uma menor necessidade de intubação do RNimediatamenteapós o parto,e uma menor necessidade de internação dos mesmos em UTI neonatal.

\section{SUMMARY}

ASSESSMENT OF FETAL MATURITY IN HIGH RISK PREGNANCIES: ANALYSIS ACCORDING TO GESTATIONAL AGE

Background. Tostudy, in high riskpregnancies, the results of fetal maturity evaluation, and analyze the perinatal results.

Methods. Between July 1998 and August 1999, 180 amniocentesiswere performed at the Fetal Surveillance Unit to assess fetal maturity. The amniotic fluid exams included Shake Test and orange cells counting. The perinatal resultswere correlated to fetal maturity in the 75 cases that delivery occurred until 7 days after the amniotic fluid analysis.

Results. The macroscopic aspects showed $91 \%$ of clear amniotic fluid $33 \%$ of hemorragic, and $3.3 \%$ of meconial fluid. The fetal maturity was evidenced at $28 \%$ of analyzed exams. When the fetus was immature, $13 \%$ of newborn require d artificial ventilation at birth and $65 \%$ were allocated at the neonatal intensive care unit. Between the mature fetuses, only one $(2.5 \%)$ required artificial ventilation at birth and $10 \%$ were allocated at the neonatal intensive care unit $(p<0.05)$. Conclusions: The fetal maturity was associated to less newborn interventions. Between the 29th and 32nd weeks $10 \%$ of amniotic fluid analysis showed maturity, providing that, when necessary, the amniotic fluid sample study can be performed at these periods. No cases of fetal maturity were diagnosed before 29th week. [Rev Ass Med Brasil 200I; 47(3): 346-5I]

KEY WORDS: Fetal maturity. Amniocentesis. Amnioticfluid.

\section{REFERÊNCIAS}

I. Zugaib M, Cha SC. Pulmão fetal. In: Zugaib $M$, Kanas $M$, editoress. Fisiologia fetal aplicada. São Paulo: Livraria Roca; 1986. p.33-59.

2. Morgenroth $\mathrm{K}$. The surfactant system of the lungs: morphology and clinical significance. Berlin: Walter de Gruyter; 1988.

3. Avery ME, Mead J. Surface properties in relation to atelectasis and hyaline membrane disease. Am J Dis Child 1959; 97:517-22.

4. Morrison J, Whybrew W, Bucovaz E. The L/S ratio and shake test in normal and abnormal pregnancies. Obstet Gynecol 1978; 52:410-4.

5. ClementsJF, Platzker ACG, Tierney DF. Assessment of the risk respiratory distress syndrome by a rapid test for surfactant in amniotic fluid. $\mathrm{N}$ Engl J Med 1972; 286:1077-8I.

6. Gluck L, Kulovich MV, Borer RC, KeidelWN. The interpretation and significance of the lecithin/sphingomyelin ratio in amniotic fluid. Am J Obstet Gynecol 1974; 120:142-55.

7. Yamamoto RM, Miyadahira S, Zugaib M. Avaliação do volume do líquido amniótico: diagnóstico ultra-sonográfico e conduta no oligoidrâmnio e poliidrâmnio. Rev Ginecol Obstet 1996; I:25-32.

8. Haidar A, Ryder TA, Wigglesworth JS. Failure of elastin development in hypoplastic lungs associated with oligohydramnios: an electronmicroscopic study. Histopathology 199I; I8:47I-3.

Artigo recebido:04/04/200।

Aceito para publicação:08/08/200I 